\title{
Light incoherence due to quantum-gravitational fluctuations of the background space
}

\author{
Michael Maziashvili: \\ Andronikashvili Institute of Physics, 6 Tamarashvili St., Tbilisi 017r, Georgia and \\ Faculty of Physics and Mathematics, Chavchavadze State University, 32 Chavchavadze Ave., Tbilisi 0179, Georgia
}

\begin{abstract}
Based on the theory of mutual coherence of light from an extended incoherent quasimonochromatic source (providing a basis of stellar interferometry) we estimate the degree of light incoherence due to quantum-gravitational fluctuations of the background metric. It is shown that the stellar interferometry observational data considered in the literature for a last few years as a manifestation against the Planck scale quantum-gravitational fluctuations of the background metric have no chance for detecting such an effect.
\end{abstract}

PACS numbers: 04.60.-m, 95.75.Kk

\section{Introduction}

Quantum gravity (QG) strongly indicates the finite resolution of space-time, that is, space-time uncertainty. A glance at a recent QG literature obviously shows that space-time uncertainty is common for all approaches to quantum gravity: space-time uncertainty relations in string theory [1, 2]; noncommutative space-time approach [3]; loop quantum gravity [4]; or space-time uncertainty relations coming from a simple Gedankenexperiments of space-time measurement [5, 6, 7, 8]. Well known entropy bounds emerging via the merging of quantum theory and general relativity also imply finite space-time resolution [9]. The combination of quantum theory and general relativity in one or another way manifests that the conventional notion of distance breaks down the latest at the Planck scale $l_{P} \simeq 10^{-33} \mathrm{~cm} \mathrm{[10]}$. Since our understanding of time is tightly related to the periodic motion along some length scale, this result implies in general an impossibility of space-time distance measurement to a better accuracy than $\sim l_{P}$. It is tantamount to say that the space-time point undergoes fluctuations of the order of $\sim l_{P}^{4}$, that is, space-time point is effectively replaced by the cell $\sim l_{P}^{4}$, we refer the reader to a very readable papers of Alden Mead [5] for his discussion regarding the status of a fundamental (minimum) length $l_{P}$, as this conceptual standpoint was unanimous in almost all subsequent papers albeit many authors apparently did not know that paper. The local fluctuations, $\sim l_{P}$, add up over the macroscopic scale $l \gg l_{P}$ in this or another way that results in fluctuation $\delta l(l)$. In view of the fact how the local fluctuations of space-time add up over the macroscopic scale different scenarios come into play. Most interesting in quantum gravity are random and holographic fluctuations. If the local fluctuations, $\sim l_{P}$, are of random nature then over the length scale $l$ they add up as $\delta l=\left(l / l_{P}\right)^{1 / 2} l_{P}[\underline{6}, 8]$. In the holographic case, the local fluctuations, $\sim l_{P}$, add up over the length scale $l$ in

*Electronic address: mishamazia@hotmail.com such a way to ensure the black hole entropy bound on the horizon region $\delta l=\left(l / l_{P}\right)^{1 / 3} l_{P}[7,[8]$. In what follows we will consider a relatively general case by parameterizing the space-time uncertainty as follows

$$
\delta l=\beta l_{P}^{\alpha} l^{1-\alpha},
$$

where $1 / 2 \leq \alpha \leq 1$ and $\beta$ is understood to be of order unity. Eq.(1) tells us that because of background metric fluctuations the length $l$ undergoes fluctuations of the order of $\delta l$. Having summarized the background metric fluctuations in Eq.(11), let us concisely formulate the problem we want to address in this paper. Metric fluctuations naturally produce the fluctuations in energymomentum of particle, for the particle with momentum $p$ has the wavelength $\lambda=2 \pi p^{-1}$ and due to length fluctuation, Eq.(11), one finds $\delta p=2 \pi \lambda^{-2} \delta \lambda, \delta E=p E^{-1} \delta p$. An interesting idea for detecting the space-time fluctuations was proposed in [11]. The idea is to consider a phase incoherence of light coming to us from extragalactic sources. The energy-momentum uncertainties result in uncertainties of phase and group velocities of the photon leading to the phase incoherence of photon during the propagation. Since the phase coherence of light from an astronomical source incident upon a two-element interferometer is necessary condition to subsequently form interference fringes, such observations offer by far the most sensitive and uncontroversial test. The interference pattern when the source is viewed through a telescope will be destroyed if the phase incoherence, $\delta \varphi$, approaches $2 \pi$. In other words, if the light with wavelength $\lambda$ received from a celestial optical source located at a distance $l$ away produces the normal interference pattern, the corresponding phase uncertainty should satisfy the condition $\delta \varphi<2 \pi$. Soon after the appearance of paper [11] it was noticed in [12] that such a naive approach overestimates the effect as the authors of [11] do not take into account the fact that the light coming from the distant stellar objects is incoherent from the beginning but acquires a mutual coherence later simply by propagation of rays over a large distance with respect to the van Cittert-Zernike theorem [13]. In order to estimate the correct size of the effect, let us follow a textbook [13], which gives the basics concerning the stellar interferometry. 


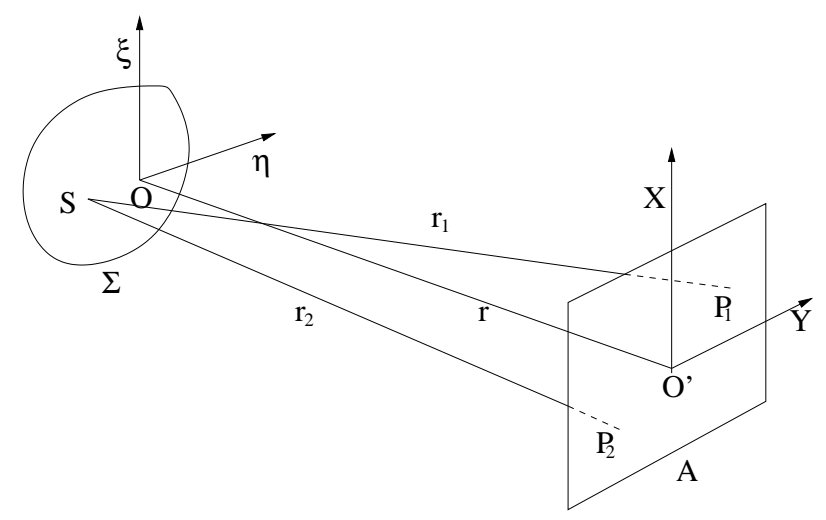

FIG. 1: A screen $A$ illuminated by an extended quasimonochromatic incoherent source $\Sigma$.

\section{Mutual coherence of light from an extended incoherent quasi-monochromatic source}

Light from a real physical source is never strictly monochromatic but rather quasi-monochromatic, even the sharpest spectral line has a finite width. In a monochromatic wave the amplitude at any point $P$ is constant, while the phase varies linearly with time, that is, a general monochromatic wave of frequency $\omega$ can be represented as a solution of the wave equation of the form

$$
\varphi(\vec{r}, t)=a(\vec{r}) e^{i[g(\vec{r})-\omega t]} .
$$

This is no longer the case in a wave produced by a real source: the amplitude and phase undergo irregular fluctuations, the rapidity of which depends on the width of spectrum $\delta \omega$,

$$
\varphi(r, t)=\int_{0}^{\infty} d \omega a(r, \omega) e^{i[k(\omega) r-\omega t]},
$$

where the amplitudes $a(r, \omega)$ differ appreciably from zero only within a narrow range around a mean frequency $\bar{\omega}$

$$
\bar{\omega}-\frac{\delta \omega}{2} \leq \omega \leq \bar{\omega}+\frac{\delta \omega}{2}, \quad \frac{\delta \omega}{\bar{\omega}} \ll 1 .
$$

Such a quasi-monochromatic wave which is usually referred to as a wave packet in the physics literature is characterized with a group and phase velocities

$$
v_{p}=\frac{\bar{\omega}}{\bar{k}}, \quad v_{g}=\left.\left(\frac{d \omega}{d k}\right)\right|_{\bar{k}} .
$$

If $\delta \omega$ is sufficiently small the wave packet Eq.(2) can be interpreted as a plane wave with frequency $\bar{\omega}$, wave number $k(\bar{\omega}) \equiv \bar{k}$ and variable amplitude

$$
A(r, t)=\int_{\bar{\omega}-\delta \omega / 2}^{\bar{\omega}+\delta \omega / 2} d \omega a(r, \omega) e^{i\{[k(\omega)-k(\bar{\omega})] r-[\omega-\bar{\omega}] t\}} .
$$

The width $\delta \omega$ determines duration of the wave packet $\delta t \simeq \delta \omega^{-1}$, an important characteristic for the interference effect. Namely, the interference effect to take place the path difference between the overlapping quasimonochromatic beams must be less than the coherence length $\delta t$. Now let us follow van Cittert-Zernike approach to the mutual coherence of light from an extended quasimonochromatic source. A screen $A$ is illuminated by an extended quasi-monochromatic incoherent source $\Sigma$ taken for simplicity to be a portion of a plane parallel to $A$, see Fig.1. The points $P_{1}$ and $P_{2}$ correspond to the slits of interferometer. We will make the following natural assumptions. The linear dimensions of $\Sigma$ are small compared to the distance $O O^{\prime}$ between the source and the screen, and the angles between $O O^{\prime}$ and the line joining a typical source point $S$ to $P_{1}$ and $P_{2}$ are small. Dividing the source into elements $d \sigma_{m}$ centered on points $S_{m}$ and denoting by $\varphi_{m 1}(t)$ and $\varphi_{m 2}(t)$ the disturbances at $P_{1}$ and $P_{2}$ due to element $d \sigma_{m}$, for total disturbances at these points one finds

$$
\varphi_{1,2}(t)=\sum_{m} \varphi_{m 1,2}(t)
$$

Correlation function between the light signals $\varphi_{1}(t)$ and $\varphi_{2}(t)$ takes the form

$\left\langle\varphi_{1}(t) \varphi_{2}^{*}(t)\right\rangle=\sum_{m}\left\langle\varphi_{m 1}(t) \varphi_{m 2}^{*}(t)\right\rangle+\sum_{m \neq n}\left\langle\varphi_{m 1}(t) \varphi_{n 2}^{*}(t)\right\rangle$

Because of mutual incoherence, the light signals coming from different elements of the source are statistically independent, that is, there is no correlation between $\varphi_{m 1}(t)$ and $\varphi_{n 2}(t)$ when $m \neq n$

$$
\left\langle\varphi_{m 1}(t) \varphi_{n 2}^{*}(t)\right\rangle=0, \quad \text { for } m \neq n .
$$

The signals $\varphi_{m 1,2}(t)$ represent spherical waves coming to the points $P_{1}$ and $P_{2}$ from the elements $d \sigma_{m}$

$$
\varphi_{m 1,2}(t)=A_{m}\left(t-r_{m 1,2}\right) \frac{e^{-i \bar{\omega}\left[t-r_{m 1,2}\right]}}{r_{m 1,2}},
$$

where $r_{m 1,2}$ denote distances between the elements $d \sigma_{m}$ and the points $P_{1,2}$. Using Eq.(3) for the correlation function one finds 


$$
\left\langle\varphi_{m 1}(t) \varphi_{m 2}^{*}(t)\right\rangle=\left\langle A_{m}\left(t-r_{m 1}\right) A_{m}^{*}\left(t-r_{m 2}\right)\right\rangle \frac{e^{i \bar{\omega}\left(r_{m 1}-r_{m 2}\right)}}{r_{m 1} r_{m 2}}=\left\langle A_{m}(t) A_{m}^{*}\left(t-r_{m 2}+r_{m 1}\right)\right\rangle \frac{e^{i \bar{\omega}\left(r_{m 1}-r_{m 2}\right)}}{r_{m 1} r_{m 2}}
$$

If the path difference $r_{m 2}-r_{m 1}$ is small compared to the coherence length (duration of the wave packet $\delta t$ ) one can neglect the retardation $r_{m 2}-r_{m 1}$ in the argument of $A^{*}$ in Eq.(41) that gives

$$
\left\langle\varphi_{1}(t) \varphi_{2}^{*}(t)\right\rangle=\sum_{m}\left\langle A_{m}(t) A_{m}^{*}(t)\right\rangle \frac{e^{i \bar{\omega}\left(r_{m 1}-r_{m 2}\right)}}{r_{m 1} r_{m 2}} .
$$

The quantity $\left\langle A_{m}(t) A_{m}^{*}(t)\right\rangle$ characterizes the intensity of the radiation from the source element $d \sigma_{m}$. So the correlation function Eq.(5) takes the form

$$
\left\langle\varphi_{1}(t) \varphi_{2}^{*}(t)\right\rangle=\int_{\Sigma} d \sigma I(\sigma) \frac{e^{i \bar{\omega}\left(r_{1}-r_{2}\right)}}{r_{1} r_{2}},
$$

where $I\left(s_{m}\right) d \sigma_{m}=\left\langle A_{m}(t) A_{m}^{*}(t)\right\rangle$. In most applications the intensity $I(\sigma)$ may be assumed to be uniform on $\Sigma$. To work out the integral Eq.(6) let us denote by $(\xi, \eta)$ the coordinates of a point $S$, referred to axes at $O$, and let $\left(x_{1}, y_{1}\right)$ and $\left(x_{2}, y_{2}\right)$ be the coordinates of $P_{1}$ and $P_{2}$ referred to parallel axes at $O^{\prime}$, see Fig.1. Retaining only leading terms in $x / r, y / r, \xi / r, \eta / r$, where $r$ is the distance $O O^{\prime}$, one finds

$r_{1}-r_{2} \approx \frac{x_{1}^{2}+y_{1}^{2}-x_{2}^{2}-y_{2}^{2}+2 \xi\left(x_{2}-x_{1}\right)+2 \eta\left(y_{2}-y_{1}\right)}{2 r}$.

Denoting

$p=\frac{x_{1}-x_{2}}{r}, q=\frac{y_{1}-y_{2}}{r}, \psi=\frac{\bar{\omega}\left(x_{1}^{2}+y_{1}^{2}-x_{2}^{2}-y_{2}^{2}\right)}{2 r}$,

the Eq.(6) takes the form

$$
\left\langle\varphi_{1}(t) \varphi_{2}^{*}(t)\right\rangle \approx \frac{e^{i \psi}}{r^{2}} \int_{\Sigma} d \xi d \eta I(\xi, \eta) e^{i \bar{\omega}(p \xi-q \eta)} .
$$

For a uniform circular source of radius $\rho$ with its center at $O$, the Eq.(7) reduces to

$$
\left\langle\varphi_{1}(t) \varphi_{2}^{*}(t)\right\rangle \sim e^{i \psi} \frac{J_{1}(v)}{v},
$$

where $v=\bar{\omega} \rho \sqrt{p^{2}+q^{2}}$ and $J_{1}$ stands for the Bessel function of the first kind and first order. In most applications the quantity $\psi$ is very small, so that one can neglect corresponding phase factor in Eq. (8). The function $J_{1}(v) / v$ decreases steadily from the value 0.5 when $v=0$ to the value zero when $v=3.83$ indicating that the degree of coherence steadily decreases and approaches complete incoherence when $P_{1}$ and $P_{2}$ are separated by the distance

$$
P_{1} P_{2}=\frac{0.61 \bar{\lambda} r}{\rho} .
$$

In experiments on interference and diffraction a departure of 12 per cent from the ideal value of coherence that occurs at $v=1$ can be taken as a maximum permissible departure that gives for the separation of points $P_{1}$ and $P_{2}$

$$
P_{1} P_{2}=\frac{0.16 \bar{\lambda} r}{\rho}
$$

\section{The degree of incoherence due to QG fluctuations}

As it was said in the introduction, due to quantumgravitational fluctuations of the background metric the length scale $l$ undergoes fluctuations of the order of $\delta l$ Eq.(11). Because of background metric fluctuations there is an increment of the wave packet width which can be simply estimated by using the Eq.(11)

$$
\delta \omega=\bar{\omega} \frac{\delta \bar{\lambda}}{\bar{\lambda}}=\bar{\omega} \beta\left(\frac{l_{P}}{\bar{\lambda}}\right)^{\alpha} .
$$

A wavelength of the light from stellar objects considered in [11, 14, 15] is in the region $\bar{\lambda} \simeq \mu \mathrm{m}$ and correspondingly for the width increment of a wave packet from Eq.(10) one finds $(1 / 2 \leq \alpha \leq 1$ and $\beta$ is of order unity)

$$
\frac{\delta \omega}{\bar{\omega}} \simeq \beta 10^{-29 \alpha} \ll 1 .
$$

In addition we need to check an important requirement that the path difference $\left|r_{m 2}-r_{m 1}\right|$ is still smaller than $\delta \omega^{-1}$. We have $\left|r_{m 2}-r_{m 1}\right| \leq P_{1} P_{2}$, where with respect to the observational data considered in [11, 14, 15], $P_{1} P_{2}$ is in the range $1 \mathrm{~m}-20 \mathrm{~m}$ (the distance between two slits of the interferometer). Recalling that $1 / 2 \leq \alpha \leq 1$ and $\beta$ takes on reasonable values, for the wavelength $\overline{\bar{\lambda}} \simeq \mu \mathrm{m}$ from Eq.(11) one finds

$$
\delta \omega^{-1} \simeq \bar{\lambda} \frac{10^{29 \alpha}}{\beta} \gg 20 \mathrm{~m}, .
$$

So one infers that there is no change in the coherence picture due to fluctuations of $\bar{\lambda}$. The observational data considered in [11, 14, 15] are collected from high-redshift astronomical sources with maximum $z=5.4$. So that the photons left the sources at the shorter wavelength $\lambda /(1+z)$. But it is easy to see that the above conclusions do not change by inclusion the factor $z=5.4$.

In light of the observational data considered in 11, 14, 15], the maximum value of $\psi$ reads

$$
\psi=\frac{\pi\left(P_{1} P_{2}\right)^{2}}{\bar{\lambda} r} \simeq 4 \cdot 10^{-11},
$$


and corresponding phase factor in Eq.(8) may evidently be neglected.

Now by taking the variations of $\rho, r$ in Eq. (9) one finds

$$
\delta\left(P_{1} P_{2}\right) \simeq \beta\left(P_{1} P_{2}\right)^{1+\alpha}\left(\frac{l_{P}}{0.16 \cdot \bar{\lambda} \cdot r}\right)^{\alpha} .
$$

Let us estimate the maximum of this variation by choosing the corresponding parameters from the data $[11,14$, 15], that is, $r \sim 1 \mathrm{kpc}, P_{1} P_{2} \sim 10^{3} \mathrm{~cm}, \bar{\lambda} \sim 10^{-4} \mathrm{~cm}$. For this set of parameters from Eq.(12) one finds

$$
\delta\left(P_{1} P_{2}\right) \sim \beta 10^{3-47 \alpha} \mathrm{cm},
$$

which for $\alpha=1 / 2$ (that gives a maximum as $1 / 2 \leq \alpha \leq$ 1) yields

$$
\delta\left(P_{1} P_{2}\right) \sim \beta 10^{-20} \mathrm{~cm}
$$

So that the observations analyzed in [11, 14, 15] have no chance to detect the quantum gravitational fluctuations described by the Eq.(11) for any reasonable value of $\beta$ and $1 / 2 \leq \alpha \leq 1$. The effect to be somewhat appreciable the ratio $\bar{\delta}\left(P_{1} P_{2}\right) / P_{1} P_{2}$ should not be highly suppressed, that is, it should be possible to control the length $P_{1} P_{2}$ with the accuracy $\delta\left(P_{1} P_{2}\right)$.

\section{Concluding remarks}

First let us outline how the problem of light incoherence from distant astronomical sources caused by the QG fluctuations of the background metric was treated in the previous study. In paper [11] the authors assumed that the light coming from the distant extragalactic sources (the diffraction/interference images of which are seen through the two slit telescopes) is coherent from the beginning, but accumulates appreciable phase incoherence if the length of propagation, $t$, is large enough. Phase incoherence accumulates at the expense of factor $t \delta \omega$ and becomes sufficiently large even for small values of $\delta \omega(\delta \omega$ is caused by the QG fluctuations of the background metric) when $t$ is large enough. So it is understood that the time dependance of the wave, $t \omega$, varies due to QG fluctuations as $\delta(t \omega)=\omega \delta t+t \delta \omega$ and because the second term is dominating it is taken as a main source of phase incoherence. The condition $t \delta \omega \geq 2 \pi$ is understood as a criterion for incoherence that should lead to the destruction of the diffraction/interference patterns when the source is viewed through a telescope. From Eq.(11) one finds $\delta \omega$ for a given $\bar{\omega}$ and then readily estimates (for a given value of $\alpha$ ) the distance $t \simeq 2 \pi / \delta \omega$.

Following this way of reasoning, in paper [14] the distance through which the wave-front recedes when the phase increases by $t \delta \omega$ is taken as an error in measurement of a length, $t$, by the light with wavelength $2 \pi / \omega$, and due to this length variation an apparent blurring of distant point sources is estimated. Those papers found the amplification of Planck scale tiny QG effect to an extent that contradicts the existing observations of diffraction/interference images through the telescopes.

In order to mitigate the situation it was suggested in [16] that the phase incoherence

$$
t \delta \omega=\omega \frac{t}{\lambda} \delta \lambda
$$

could be reduced by taking the factor $(l / \lambda)^{1-\alpha}$ instead of $l / \lambda$ as it stands in Eq.(15). For motivating this step, the authors of paper [16] were assuming that in the case $\alpha=1 / 2$ the fluctuations $\delta \lambda$ were expected to take on \pm sign with equal probability and respectively had to add up over the length scale $t$ by the cumulative factor $(l / \lambda)^{1 / 2}$. Then for other values of $\alpha$, the cumulative factor was assumed of the form $(l / \lambda)^{1-\alpha}$. (This reduced expression for the phase incoherence is used in [15] as well.) First, one may question that as in the particular case $\alpha=1 / 2$ the local fluctuations $\sim l_{P}$ add up over the macroscopic scale $l$ to $\delta l=\beta\left(l / l_{P}\right)^{1 / 2} l_{P}$ that obviously contradicts the argument that $\delta \lambda$ should also add by the same cumulative factor. Second, what is most important, this artificial trick has nothing to do with the physics behind the phenomenon under consideration. As we see the approaches of these papers to the problem of stellar interferometry are oversimplified that leads to the misleading results. The ideology of papers [11, 14, 15, 16] has little to do with the theory of mutual coherence of light from an extended incoherent quasi-monochromatic source, that may be the only reliable way for a proper understanding of question under consideration.

The discussion of our paper is based on the theory of mutual coherence of light from an extended incoherent quasi-monochromatic source (which provides the basis of stellar interferometry). What we learn from this theory is that the light coming from a real astronomical source has a natural finite width $\delta \omega$ from the very outset and the contribution to the width due to QG fluctuations is negligible. Moreover, even were it not so this would not affect the mutual coherence of light as long as $\delta \omega$ satisfies the conditions

$$
\frac{\delta \omega}{\bar{\omega}} \ll 1, \quad\left|r_{m 1}-r_{m 2}\right| \ll \delta \omega^{-1} .
$$

The rate of QG incoherence is discouragingly small as it is demonstrated by the Eqs.(13, 14), to be detectable by the stellar interferometry observations considered in 11, 14, 15, 16.

\section{Acknowledgments}

Author is indebted to Professors Jean-Marie Frère and Peter Tinyakov for invitation and hospitality at the Service de Physique Théorique, Université Libre de Bruxelles, where a great deal of this paper was done. Special thanks are due to Peter Tinyakov for many useful discussions and comments. The work was supported 
in part by the INTAS Fellowship for Young Scientists; CRDF/GRDF grant and the Georgian President Fellow- ship for Young Scientists.
[1] G. Veneziano, Europhys. Lett. 2 (1986) 199; D. J. Gross and P. F. Mende, Nucl. Phys. B303 (1988) 407;

D. Amati, M. Ciafaloni and G. Veneziano, Phys. Lett. B216 (1989) 41;

K. Konishi, G. Paffuti, P. Provero, Phys. Lett. B234 (1990) 276;

R. Guida, K. Konishi and P. Provero, Mod. Phys. Lett. A6 (1991) 1487.

[2] M. Li and T. Yoneya, Phys. Rev. Lett. 78 (1997) 1219, hep-th/9611072.

T. Yoneya, Prog. Theor. Phys. 103 (2000) 1081, hep-th/0004074.

T. Yoneya, Prog. Theor. Phys. Suppl. 171 (2007) 87.

[3] D. Ahluwalia, Phys. Lett. B339 (1994) 301, gr-qc/9308007

M. Maggiore, Phys. Lett. B304 (1993) 65, hep-th/9301067

M. Maggiore, Phys. Rev. D49 (1994) 5182, hep-th/9305163

M. Maggiore, Phys. Lett. B319 (1993) 83, hep-th/9309034

S. Doplicher, K. Fredenhagen and J. E. Roberts, Commun. Math. Phys. 172 (1995) 187, hep-th/0303037;

N. Seiberg and E. Witten, JHEP 9909 (1999) 032, hep-th/9908142

[4] A. Ashtekar and J. Lewandowski, Class. Quant. Grav. 21, (2004) R53;

C. Rovelli, Quantum Gravity, (Cambridge University Press, Cambridge 2004);

T. Thiemann, Introduction to Modern Canonical Quantum General Relativity, (Cambridge University Press, Cambridge, 2007).

[5] C. Alden Mead, Phys. Rev. 135 (1964) B849;

C. Alden Mead, Phys. Rev. 143 (1966) 990;

T. Padmanabhan, Class. Quant. Grav. 4 (1987) L107;

G. Amelino-Camelia, Mod. Phys. Lett. A9 (1994) 3415, gr-qc/9603014

F. Scardigli, Phys. Lett. B452 (1999) 39, hep-th/9904025

R. Adler and D. Santiago, Mod. Phys. Lett. A14 (1999) 1371, gr-qc/9904026.

[6] G. Amelino-Camelia, Lect. Notes Phys. 541 (2000) 1, gr-qc/9910089.

[7] F. Károlyházy, Nuovo Cim. A42 (1966) 390;

F. Károlyházy, A. Frenkel and B. Lukács, in Physics as Natural Philosophy (Eds. A. Shimony and H. Feschbach, MIT Press, Cambridge, MA, 1982);

F. Károlyházy, A. Frenkel and B. Lukács, in Quan- tum Concepts in Space and Time (Eds. R. Penrose and C. J. Isham, Clarendon Press, Oxford, 1986);

Y. J. Ng and H. van Dam, Mod. Phys. Lett. A9 (1994) 335 ;

N. Sasakura, Prog. Theor. Phys. 102 (1999) 169, hep-th/9903146

M. Maziashvili, Int. J. Mod. Phys. D16 (2007) 1531, gr-qc/0612110

[8] M. Maziashvili, Int. J. Mod. Phys. A23 (2008) 1747, arXiv: 0709.0898 [gr-qc].

[9] J. D. Bekenstein, Phys. Rev. D23 (1981) 287;

J. D. Bekenstein, Phys. Rev. D49 (1994) 1912, gr-qc/9307035

R. Bousso, JHEP 9907 (1999) 004, hep-th/9905177.

E. E. Flanagan, D. Marolf and R. M. Wald, Phys. Rev. D62 (2000) 084035, hep-th/9908070

R. Bousso, Rev. Mod. Phys. 74 (2002) 825, hep-th/0203101,

R. Bousso, E. E. Flanagan and D. Marolf, Phys. Rev. D68 (2003) 064001, hep-th/0305149

[10] L. J. Garay, Int. J. Mod. Phys. A10 (1995) 145, gr-qc/9403008

[11] R. Lieu and L. Hillman, astro-ph/0211402

R. Lieu and L. Hillman, Astrophys. J. 585 (2003) L77, astro-ph/0301184

[12] D. H. Coule, Class. Quant. Grav. 20 (2003) 3107, astro-ph/0302333

[13] P. H. van Cittert, Physica 1 (1934) 201;

P. H. van Cittert, Physica 6 (1939) 1129;

F. Zernike, Physica 5 (1938) 785;

L. Janossy, Nuovo Cimento 6 (1957) 111;

L. Janossy, Nuovo Cimento 12 (1959) 369;

H. H. Hopkins, Proc. Roy. Soc. (London) A208 (1951) 263 ;

H. H. Hopkins, Proc. Roy. Soc. (London) A217 (1953) 408;

M. Born and E. Wolf, Principles of Optics, (Cambridge University Press, 2002).

[14] R. Ragazzoni, M. Turatto and W. Gaessler, Astrophys. J. 587 (2003) L1, astro-ph/0303043.

[15] E. Steinbring, Astrophys. J. 655 (2007) 714, astro-ph/0610422

[16] Y. Jack Ng, H. van Dam and W. A. Christiansen, Astrophys. J. 591 (2003) L87, astro-ph/0302372.

W. A. Christiansen, Y. Jack Ng and H. van Dam, Phys. Rev. Lett. 96 (2006) 051301, gr-qc/0508121 